\title{
ACKNOWLEDGEMENTS
}

The expert workshop from which these papers are drawn was funded by the Better World Fund of the United Nations Foundation and I wish to acknowledge this support and in particular to thank Reid Detchon for his calm and unwavering backing throughout its long gestation.

The project was initiated in 2002, at the instigation of Dr. Robert Watson, then Chairman of the IPCC, for whose early appreciation of the potential of linking bio-energy to carbon capture and sequestration I am grateful.

Throughout the later stages of preparation, and in subsequent editorial work, I have been helped by Edward Y. Sumoto, who accepted my offer of an internship with this project on completion of his studies at Georgetown University. Without him I do not believe the workshop would have happened, and I cannot express the debt I owe to his energy and reliability in keeping the project moving. To him goes the credit for our website, www.accstrategy.org, where the papers in this special issue have been posted (in original authors' format) since July 2005, along with other papers that have been held over for publication in regular issues of this Journal.

Finally, thanks are due to the authors of all these papers, to distinguished discussants, and to the participants in the workshop, whose expertise and commitment provided the basis for a climate change strategy that could better prepare the world for a sudden worsening of the global climate threat.

\section{PETER READ}

18.vii.05

Massey University

Palmerston North

New Zealand

E-mail: p.read@massey.ac.nz

\section{Note}

In this Special Issue the term "bio-char" is used for the carbon-rich and highly porous material produced from the pyrolysis of biomass. This is in lieu of the commonly used misnomer 'charcoal' for a material that has nothing to do with coal and is most familiar in the form derived by slow pyrolysis of wood but which can be derived, in varying quality, from slow or fast pyrolysis of any biomass raw material. This usage is noted again in those Articles where bio-char has an important role. 